TRANSACTIONS OF THE

AMERICAN MATHEMATICAL SOCIETY

Volume 349, Number 8, August 1997, Pages 3321-3342

S 0002-9947(97)01966-1

\title{
STRICT DEFINITENESS OF INTEGRALS VIA COMPLETE MONOTONICITY OF DERIVATIVES
}

\author{
L. MATTNER
}

Dedicated with gratitude to Professor Erwin Mues, on the occasion of his sixtieth birthday

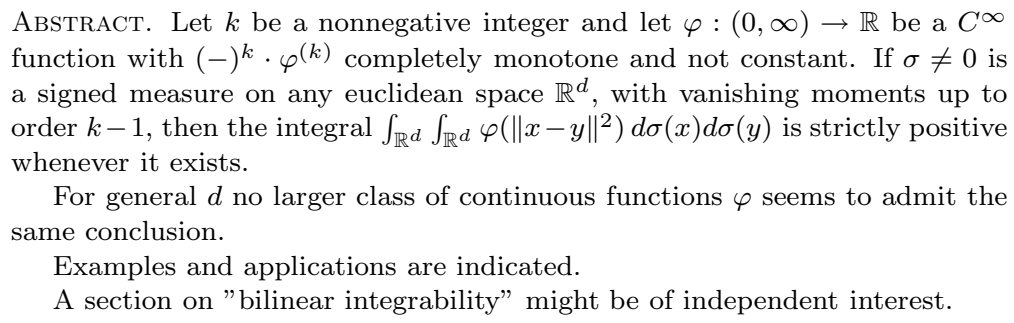

\section{INTRODUCTION}

This paper considers inequalities for integrals of the form

$$
I(\varphi ; \sigma, \tau):=\int_{\mathbb{R}^{d}} \int_{\mathbb{R}^{d}} \varphi\left(\|x-y\|^{2}\right) d \sigma(x) d \tau(y)
$$

for certain functions $\varphi:[0, \infty) \rightarrow(-\infty, \infty]$ and signed Radon measures $\sigma$ and $\tau$, where $\|\cdot\|$ denotes the euclidean norm in any dimension $d$. The emphasis is on strict inequalites, in particular strict positive definiteness of the quadratic form

$$
\sigma \mapsto I(\varphi ; \sigma, \sigma)
$$

on suitable subspaces of the set of all signed measures.

In order to see what kind of functions $\varphi$ one should consider in this connection, let us first restrict attention to $\varphi$ real-valued and continuous on $[0, \infty)$ and $\sigma$ molecular (i.e., $\sigma$ is a finite linear combination of Dirac measures). Fix a $k \in \mathbb{N}_{0}$, the set of all nonnegative integers. In this case Miccheli [20, Theorem 2.2 and Remark 3.3] has shown that the inequality (7) below holds if $\sigma \neq 0$ obeys the moment conditions (6) and $\varphi$ is $C^{\infty}$ on $(0, \infty)$ with $(-)^{k} \varphi^{(k)}$ completely monotone and nonconstant. He also claimed, with an indication of a method of proof, that no other real-valued continuous functions $\varphi$ on $[0, \infty)$ admit the same conclusion for every dimension d. (If in (7) " $<$ " is weakened to " $\leq$ ", then the only additional functions admitting the weakened conclusion are the polynomials of degree $\leq k$.) Guo, $\mathrm{Hu}$ and Sun [11] offered a proof of Micchelli's claim. These results are generalizations of the

Received by the editors January 28, 1996.

1991 Mathematics Subject Classification. Primary 26D15, 43A35, 31A15, 60E15.

Key words and phrases. Bernstein functions, Besicovitch covering theorem, bilinear integrability, conditionally positive definite functions, determinate moment problem, energy integrals, integral inequalities, logarithmic potential theory, moment inequalities, radial analysis. 
special cases $k=0$ and $k=1$ obtained by Schoenberg [26, Theorems $3,3^{\prime}, 6^{\prime}$ ], the case $k=1$ being somewhat implicit. They belong to the theory of positive definite functions on semigroups, for which Berg, Christensen and Ressel [2] and Sasvári [25] are useful references.

The purpose of the present paper is to extend the implication $(6) \Rightarrow(7)$ below to arbitrary (i.e., not necessarily molecular) signed measures $\sigma$, subject only to the condition that the l. h. s. of (7) is defined. In this setting it is then natural to allow, more generally, functions $\varphi$ which may have a singularity at zero. (An example, fulfilling the conditions of Theorem 2.2 and its corollaries stated below, with $k=1$, is given by $\varphi(t)=-\log t$.) The principal difficulty in this twofold extension is to avoid any approximation argument which might weaken " $<$ " in (7) to " $\leq$ ". This difficulty is finally resolved by using the determinateness of a certain moment problem - see the proposition 6.1 in Section 6 .

The author conjectures that Theorem 2.2 and its corollaries are optimal in the sense that no continuous $(-\infty, \infty]$-valued functions $\varphi$ with $\left.\varphi\right|_{(0, \infty)}$ finite valued other than the considered ones obey the corresponding conclusion for every dimension $d$. This conjecture is known to be true, from the above-mentioned paper [11], if it is restricted to functions finite valued also at zero.

The rest of this paper is structured as follows. Section 2 contains the necessary definitions and statements of our main results. Section 3 discusses briefly some examples. Lemmas concerning the classes $\mathcal{B}_{k}$ are collected in Section 4. Section 5, for want of a better name entitled Bilinear integrability, presents some auxiliary results on the apparantly not so trivial question as to whether a double integral $\iint \psi d \mu d \nu$ with $\psi, \mu, \nu \geq 0$ is necessarily finite when it is known that $\iint \psi d \mu d \mu$ and $\iint \psi d \nu d \nu$ are both finite. Finally, Section 6 contains the proofs of our main results.

\section{MAin RESUlts}

2.1. Notation etc. Recall that a function $\psi:(0, \infty) \rightarrow \mathbb{R}$ is called completely monotone iff it is $C^{\infty}$ with $(-)^{n} \psi^{(n)} \geq 0$ for every $n \in \mathbb{N}_{0}$. Throughout this paper, $k$ denotes an element of $\mathbb{N}_{0}$. Consider any $C^{\infty}$ function $\varphi:(0, \infty) \rightarrow \mathbb{R}$ such that $(-)^{k} \varphi^{(k)}$ is completely monotone on $(0, \infty)$. By the elementary lemma 4.5 below, $\varphi$ extends by continuity to a $(-\infty, \infty]$-valued function on $[0, \infty)$. The set of all functions obtained in this manner will be denoted by $\mathcal{B}_{k}$. In case $k=0$, the assumption $\varphi \in \mathcal{B}_{k} \backslash \mathcal{B}_{k-1}$ is to be read as $\varphi \in \mathcal{B}_{0}$. The letter $\mathcal{B}$ refers to S. Bernstein; see Bernstein's theorem in Section 4 below. That section collects properties of these functions. Examples are given in Subsection 3.1. For the time being, note that obviously $\mathcal{B}_{0} \subset \mathcal{B}_{1} \subset \ldots$.

Since we treat possibly divergent integrals, we have to be quite explicit about our conventions concerning measures and integrals. We let $M_{+}\left(\mathbb{R}^{d}\right)$ denote the set of all nonnegative Radon measures on $\mathbb{R}^{d}$. So $\mu \in M_{+}\left(\mathbb{R}^{d}\right)$ iff $\mu$ is a nonnegative measure on the $\sigma$-algebra of all Borel-sets of $\mathbb{R}^{d}$ and $\mu$ is finite-valued on compact sets. $M\left(\mathbb{R}^{d}\right)$ denotes the set of all signed, not necessarily bounded Radon measures on $\mathbb{R}^{d}$. So $\sigma \in M\left(\mathbb{R}^{d}\right)$ iff $\sigma$ is a real-valued $\sigma$-additive set function on the $\delta$ ring of all bounded Borel sets of $\mathbb{R}^{d}$. We may identify $M_{+}\left(\mathbb{R}^{d}\right)$ with the subset of all nonnegative elements of $M\left(\mathbb{R}^{d}\right)$, so that every $\sigma \in M\left(\mathbb{R}^{d}\right)$ admits a Jordan decomposition $\sigma=\sigma_{+}-\sigma_{-}$with $\sigma_{+}, \sigma_{-} \in M_{+}\left(\mathbb{R}^{d}\right)$ minimal. 
If $\sigma \in M\left(\mathbb{R}^{d}\right)$ and if $f=f_{+}-f_{-}$is a $[-\infty, \infty]$-valued measurable function on $\mathbb{R}^{d}$, then we say that $\int f d \sigma$ exists, and define

$$
\int f d \sigma:=\left(\int f_{+} d \sigma_{+}+\int f_{-} d \sigma_{-}\right)-\left(\int f_{+} d \sigma_{-}+\int f_{-} d \sigma_{+}\right),
$$

whenever the r.h.s. is not of the form $\infty-\infty$. In the case of the double integrals (1), we have $(\sigma \otimes \tau)_{+}=\sigma_{+} \otimes \tau_{+}+\sigma_{-} \otimes \tau_{-}$and $(\sigma \otimes \tau)_{-}=\sigma_{+} \otimes \tau_{-}+\sigma_{-} \otimes \tau_{+}$, so that (2) reads

$$
\begin{aligned}
I(\varphi ; \sigma, \tau):=( & \left.I\left(\varphi_{+} ; \sigma_{+}, \tau_{+}\right)+I\left(\varphi_{+} ; \sigma_{-}, \tau_{-}\right)+I\left(\varphi_{-} ; \sigma_{+}, \tau_{-}\right)+I\left(\varphi_{-} ; \sigma_{-}, \tau_{+}\right)\right) \\
& -\left(I\left(\varphi_{-} ; \sigma_{+}, \tau_{+}\right)+I\left(\varphi_{-} ; \sigma_{-}, \tau_{-}\right)+I\left(\varphi_{+} ; \sigma_{-}, \tau_{+}\right)+I\left(\varphi_{+} ; \sigma_{+}, \tau_{-}\right)\right),
\end{aligned}
$$

whenever the r.h.s. is not of the form $\infty-\infty$.

$d \in \mathbb{N}$ denotes the dimension of the underlying space $\mathbb{R}^{d}$. We use standard notations for multiindices $\alpha \in \mathbb{N}_{0}^{d}$, such as $x^{\alpha}:=x_{1}^{\alpha_{1}} \cdot \ldots \cdot x_{d}^{\alpha_{d}},|\alpha|:=\alpha_{1}+\ldots+\alpha_{d}$, $\alpha !:=\alpha_{1} ! \cdot \ldots \cdot \alpha_{d} !$, and $\left(\begin{array}{l}l \\ \alpha\end{array}\right):=\frac{l !}{\alpha !}$.

Except in Section $5,\|\cdot\|$ always denotes the euclidean norm on $\mathbb{R}^{d}$.

2.2. Theorem. Let $\varphi \in \mathcal{B}_{k}$ and let $\mu, \nu \in M_{+}\left(\mathbb{R}^{d}\right)$ satisfy

$$
\int_{\mathbb{R}^{d}} x^{\alpha} d \mu(x)=\int_{\mathbb{R}^{d}} x^{\alpha} d \nu(x) \neq \pm \infty \quad(|\alpha| \leq k-1) .
$$

Then

$$
2 I(\varphi ; \mu, \nu) \leq I(\varphi ; \mu, \mu)+I(\varphi ; \nu, \nu)
$$

whenever the r. h. s. in (5) exists.

If the r. h. s. in (5) is finite, then the l. h. s. is finite as well. In this case equality occurs iff $\mu=\nu$, or $(-)^{k} \varphi^{(k)}=0$, or $(-)^{k} \varphi^{(k)}$ is constant and also $\int x^{\alpha} d \mu(x)=$ $\int x^{\alpha} d \nu(x) \neq \pm \infty$ for $|\alpha|=k$.

2.3. Remarks. a) In checking the existence or finiteness of either side in (5), the propositions 4.6, 4.7, 5.1, 5.2 and 5.3 given below may be helpful.

For example, if $\varphi \in \mathcal{B}_{k}$ and $\mu, \nu \in M_{+}\left(\mathbb{R}^{d}\right)$ with $\int\|x\|^{2 k} d(\mu+\nu)(x)<\infty$, then $\mu$ and $\nu$ are bounded and, by 4.6 and $4.7, \int(-)^{k} \cdot \varphi_{B}\left(\|x\|^{2}\right) d(\mu+\nu)(x)<\infty$, so that (23) of 4.7 and 5.2 imply $I\left((-)^{k} \cdot \varphi_{B} ; \mu, \nu\right)<\infty$; hence $I(\varphi ; \mu, \nu) \in(-\infty, \infty]$.

b) Let $\varphi \in \mathcal{B}_{k} \backslash \mathcal{B}_{k-1}$ and $\mu, \nu \in M_{+}\left(\mathbb{R}^{d}\right)$. If $k$ is odd, then it may happen that $I(\varphi ; \mu, \nu)=-\infty$ and that the r.h.s. of (5) is undefined, namely $I\left(\varphi_{-} ; \mu, \mu\right)=$ $I\left(\varphi_{+} ; \mu, \mu\right)=\infty$. See 6.4 for an example.

c) In the corollaries below we include the assumption " $(-)^{k} \varphi^{(k)}$ nonconstant" only in order to avoid tedious repetitions of the discussion of equality.

2.4. Corollary. Let $\varphi \in \mathcal{B}_{k}$ with $(-)^{k} \varphi^{(k)}$ nonconstant, and let $\sigma \in M\left(\mathbb{R}^{d}\right), \sigma \neq 0$, satisfy

$$
\int_{\mathbb{R}^{d}} x^{\alpha} d \sigma(x)=0 \quad(|\alpha| \leq k-1) .
$$

Then

$$
\int_{\mathbb{R}^{d}} \int_{\mathbb{R}^{d}} \varphi\left(\|x-y\|^{2}\right) d \sigma(x) d \sigma(y) \in(0, \infty]
$$

whenever the l. h. s. exists. 
2.5. Corollary. Let $\varphi \in \mathcal{B}_{k} \backslash \mathcal{B}_{k-1}$ with $(-)^{k} \varphi^{(k)}$ nonconstant. Then

$$
M^{\varphi}\left(\mathbb{R}^{d}\right):=\left\{\sigma \in M\left(\mathbb{R}^{d}\right): I(|\varphi| ;|\sigma|,|\sigma|)<\infty\right\}
$$

is a vector subspace of $M\left(\mathbb{R}^{d}\right)$, and when $k \geq 1$

$$
\int\|x\|^{2(k-1)}|d \sigma(x)|<\infty \quad\left(\sigma \in M^{\varphi}\left(\mathbb{R}^{d}\right)\right)
$$

The bilinear form

$$
(\sigma, \tau) \mapsto I(\varphi ; \sigma, \tau) \quad\left(\sigma, \tau \in M^{\varphi}\left(\mathbb{R}^{d}\right)\right)
$$

is well-defined, real-valued, and strictly positive definite on the subspace

$$
\left\{\sigma \in M^{\varphi}\left(\mathbb{R}^{d}\right): \int x^{\alpha} d \sigma(x)=0 \quad(|\alpha| \leq k-1)\right\} .
$$

2.6. Corollary. Let $\varphi \in \mathcal{B}_{k}$ with $(-)^{k} \varphi^{(k)}$ nonconstant. Let $\left(c_{\alpha}: \alpha \in \mathbb{N}_{0}^{d},|\alpha| \leq\right.$ $k-1)$ be a system of real numbers. Then

$$
\sigma \mapsto I(\varphi ; \sigma, \sigma)
$$

is a strictly convex function on the convex set

$$
\left\{\sigma \in M^{\varphi}\left(\mathbb{R}^{d}\right): \int x^{\alpha} d \sigma(x)=c_{\alpha} \quad(|\alpha| \leq k-1)\right\} .
$$

\section{EXAMPLES AND APPLICATIONS}

3.1. Examples of $\mathcal{B}_{k}$-functions. As the lemma 4.3 in Section 4 below makes precise, the most basic examples of $\mathcal{B}_{k}$-functions are given by the monomials $(t \mapsto$ $\left.(-t)^{k}\right) \in \mathcal{B}_{k} \backslash \mathcal{B}_{k-1}$ and the exponential $\left(t \mapsto e^{-t}\right) \in \mathcal{B}_{0}$.

For polynomial functions $\varphi$ we have the implications

$$
\varphi \in \mathcal{B}_{k} \Rightarrow \operatorname{deg} \varphi \leq k \Rightarrow \varphi \in \mathcal{B}_{k+1} .
$$

(To prove the first, consider large values of the variable $t$.)

Further obvious examples are of the form $\pm t^{\alpha}$. More precisely, let $\varepsilon \in\{-1,1\}$ and $\alpha \in \mathbb{R}$. Then it is easily checked that

$$
\left(t \mapsto \varepsilon \cdot t^{\alpha}\right) \in\left\{\begin{array}{cl}
\mathcal{B}_{0} & \text { iff } \alpha \leq 0 \text { and } \varepsilon=1, \\
\mathcal{B}_{k} \backslash \mathcal{B}_{k-1} \text { with } k \in \mathbb{N} & \text { iff } k-1 \leq \alpha \leq k \text { and } \varepsilon=(-)^{k} .
\end{array}\right.
$$

It is also obvious that $-\log \in \mathcal{B}_{1} \backslash \mathcal{B}_{0}$. More generally, the formula, easily checked by induction,

$$
\left(t^{\alpha} \cdot \log t\right)^{(n)}=\left(\sum_{j=0}^{n-1} \frac{(\alpha)_{n}}{\alpha-j}+(\alpha)_{n} \cdot \log t\right) \cdot t^{\alpha-n},
$$

where $(\alpha)_{n}:=\alpha \cdot(\alpha-1) \cdot \ldots \cdot(\alpha-n+1)$, shows that $\left(t \mapsto \varepsilon \cdot t^{\alpha} \cdot \log t\right)$ belongs to $\mathcal{B}_{k} \backslash \mathcal{B}_{k-1}$ iff $k=\alpha+1 \geq 1$ and $\varepsilon=(-)^{k}$.

3.2. The function $\varphi(t)=\varepsilon \cdot t^{\beta / 2}$.

3.2.1. The trivial case $\beta \leq-d, \varepsilon=1$. In this case, $\varphi \in \mathcal{B}_{0}$. But if $\mu \in M_{+}\left(\mathbb{R}^{d}\right)$ is not the zero measure, then $I(\varphi ; \mu, \mu)=\infty$. This follows from 5.3, since (38) is not true for $\psi(r)=r^{\beta}$. Thus Theorem 2.2 and its corollaries, while applicable with $k=0$, yield only trivial results. (But our proof of triviality appears to be nontrivial.) 
3.2.2. The case $\beta \in(-d, 0], \varepsilon=1, k=0$ : potential theories. In this case, $\varphi \in \mathcal{B}_{0}$.

If $\beta=0$, then $\varphi \equiv 1$, and Theorem 2.2 merely states the inequality of the geometric and artihmetic means for the numbers $\mu\left(\mathbb{R}^{d}\right), \nu\left(\mathbb{R}^{d}\right) \in[0, \infty]$, with a correct discussion of equality.

Now consider the nontrivial case $\beta \in(-d, 0)$. Then $\varphi$ is a nonconstant $\mathcal{B}_{0}$ function. It is customary to write

$$
\varphi\left(\|x-y\|^{2}\right)=\frac{1}{\|x-y\|^{d-\alpha}} \quad\left(x, y \in \mathbb{R}^{d}\right)
$$

with $\alpha=d+\beta \in(0, d): \varphi\left(\|x-y\|^{2}\right)$ is the so-called M. Riesz kernel of order $\alpha$ considered in potential theory; see Landkof [15]. In that context, $I(\varphi ; \sigma, \tau)$ is called the mutual energy of $\sigma$ and $\tau$. For $d \geq 3$ and $\alpha=2$, and in particular for $d=3$, we get the classical Newton kernel.

Corollary 2.5 states that the set $M^{\varphi}\left(\mathbb{R}^{d}\right)$ of all signed measures with finite energy is a pre-Hilbert space, with respect to the mutual energy as inner product.

This is of course known, but far from being trivial. See [15, p. 79] for the classical proof, relying on the uniqueness theorem for Riesz potentials of index $\frac{\alpha}{2}$, [15, p. 74]. One may also consult [17, p. 146] for an alternative proof of the latter.

Observe that even the fact that $M^{\varphi}\left(\mathbb{R}^{d}\right)$ is a vector space is considered to be deep in [7, p. 227] (in a partly more restrictive $(\alpha=2)$ and partly more general (Green function of an arbitrary region instead of $\mathbb{R}^{d}$ ) context). In our proof of Corollary 2.5, this is deduced from the proposition 5.3 below, using the antitonicity of $\varphi\left(t^{2}\right)$ only. In this connection see also the discussion on logarithmic potential theory below.

3.2.3. The case $\beta \in(0,2], \varepsilon=-1, k=1$ : extremal problems for probability distributions. In this case, $\varphi \in \mathcal{B}_{1} \backslash \mathcal{B}_{0}$. Let $X$ and $Y$ be two $\mathbb{R}^{d}$-valued random vectors, independent and identically distributed.

How large can the expected euclidean distance $E[\|X-Y\|]$ be if we know that $E\left[\|X\|^{2}\right]=1$ ? This question was asked for two different reasons and solved with two different methods in Mattner [16], [18] and Buja, Logan, Reeds \& Shepp [6]. For example, if $d \geq 3$, then the unique maximizing distribution for $X$ is the uniform distribution on the unit sphere. Both proofs of this result used the fact that the problem under consideration is a convex minimization problem for the distribution $\sigma$ of $X$. The said fact follows from Corollary 2.6 applied to the present $\varphi$, with $\beta=1, k=1, c_{0}=1$.

For another illustration of the same idea, let $\beta \in(0,2)$, assume $E\left[\|X\|^{\beta}\right]<\infty$, and let $T$ be any orthogonal map on $\mathbb{R}^{d}$. We claim that

$$
E\left[\|X-Y\|^{\beta}\right] \leq E\left[\|X-T Y\|^{\beta}\right]
$$

holds, with equality occuring iff $X$ and $T X$ have the same distribution. (This is a generalization and discussion of equality of $[6,(2.1)$ and Theorem 2.3 , the case $p=2$ ]. The elementary case $d=1, \beta=1$ occurs in Kallenberg [14].) Readers trying to understand (11) intuitively are refered to (13) and 3.2. $\infty$ below. To prove (11), let $\mu$ denote the distribution of $X$ and $\mu^{T}$ that of $T X$. Then $\sigma:=\mu-\mu^{T}$ satisfies $\sigma\left(\mathbb{R}^{d}\right)=0$, so that Corollary 2.4 and $\|T x-T y\|=\|x-y\|$ yield

$$
0 \leq \frac{1}{2} I(\varphi ; \sigma, \sigma)=I(\varphi ; \mu, \mu)-I\left(\varphi ; \mu, \mu^{T}\right)
$$

with equality iff $\mu=\mu^{T}$. 
3.2.4. The case $\beta \in(2,4], \varepsilon=1, k=2$ : probability distributions with given expectations. In this case, $\varphi \in \mathcal{B}_{2} \backslash \mathcal{B}_{1}$. Let $X$ and $Y$ again denote independent and identically distributed $\mathbb{R}^{d}$-valued random vectors, but assume

$$
E[X]=0
$$

from the outset.

What can we say about $E\left[\|X-Y\|^{3}\right]$ if some moments of $\|X\|$, e.g. $E\left[\|X\|^{3}\right]$, are given? This question is of interest in connection with the the derivation of sharp error bounds in the central limit theorem; see, e.g., Esseen [8] and Bhattacharya \& Ranga Rao [4, p. 66, Lemma 8.8], where, however, only the case $d=1$ is treated. By Corollary 2.6 and assumption (12), we now know that $E\left[\|X-Y\|^{3}\right]$ is a strictly convex functional of the distribution of $X$. This makes the method of Mattner [16], [18] applicable, which the author intends to use to give a more complete solution of the problem in a separate paper.

It also becomes easy to push the investigations of Polya \& Szegö [21], Björck [5] and Alexander \& Stolarsky [1] a bit further. For example, the following complement to [5, Theorem 7] is an easy consequence of Corollary 2.6: If $\beta \in(2,4)$ and if $(12)$ and $\|X\| \geq 1$ hold almost surely, then the unique distribution for $X$ minimizing $E\left[\|X-Y\|^{\beta}\right]$ is the uniform distribution on the unit sphere. (First derive orthogonal invariance, then project onto the sphere.)

With a view at (11), let $\beta \in(2,4)$, assume $E\left[\|X\|^{\beta}\right]<\infty$ in addition to (12), and again let $T$ denote any orthogonal map on $\mathbb{R}^{d}$. Now we have

$$
E\left[\|X-Y\|^{\beta}\right] \geq E\left[\|X-T Y\|^{\beta}\right],
$$

with equality occurring iff $X$ and $T X$ have the same distribution. (The case $d=1$ of this proposition is due to $[8,(2.7)$ and $(5.1)])$. This may be deduced from Corollary 2.4 analogously to (11), since $\sigma$ now has vanishing first moments.

3.2. $\infty$. A curiosity. There exist independent and identically distributed $\mathbb{R}$-valued random variables $X$ and $Y$ such that

$$
E\left[|X-Y|^{\beta}\right]-E\left[|X+Y|^{\beta}\right]
$$

is nonzero and has the sign $(-)^{k+1}$ whenever $\beta \in(2 k, 2 k+2)$ with $k \in \mathbb{N}_{0}$.

For a proof, choose $X$ asymmetrically distributed with all odd moments vanishing. Then apply Corollary 2.4 as in 3.2.3 and 3.2.4, with $T X=-X$, using the fact that all moments of $\sigma \neq 0$ vanish.

3.3. The function $\varphi(t)=\frac{1}{2} \log \frac{1}{t}, k=1$ : logarithmic potential theory. This $\varphi$ is a nonconstant function belonging to $\mathcal{B}_{1} \backslash \mathcal{B}_{0}$. So, if $d \in \mathbb{N}$ and $\sigma \in M\left(\mathbb{R}^{d}\right)$ with $\sigma\left(\mathbb{R}^{d}\right)=0$, then Corollary 2.4 yields

$$
\iint \log \frac{1}{\|x-y\|} d \sigma(x) d \sigma(y) \in(0, \infty]
$$

whenever the l.h.s. is defined. This result is usually stated under the superfluous assumption that $\sigma$ is compactly supported, and sometimes for $d=2$ only. The original proof is due to $[10$, p. 61$]$. Other proofs may be found in $[15$, p. 76$]$, [7, p. $248]$ and $[12$, p. 281]. Of these four references, Doob [7] is the only one stating the vector space property of our $M^{\varphi}\left(\mathbb{R}^{d}\right)$, albeit for the subspace defined by (6) only. This property seems to be taken for granted in the other three references, e.g. in 
[12, equation (16.4.12)]. Accordingly, a reference to our proposition 5.3, once with $\varphi(t)=\left(\log \frac{1}{t}\right)_{+}$and once with $\varphi(t)=(\log t)_{+}$, would seem to fill a gap in these works, while our example 5.5 shows that the vector space property is not completely trivial.

\section{Functions With SOME DeRivative COMPletely MONOTONE}

This section collects properties of $\mathcal{B}_{k}$ functions, as defined in the first paragraph of Section 2. Since that definition refers to the lemma 4.5 below, we use the symbol $\mathcal{B}_{k}$ only from 4.6 onwards, with the exception of some earlier subsection headings.

It is true that the facts presented in this section are either rather elementary or else minor variations of well-known ideas. Our excuse for presenting them in detail here is that we have to refer to them frequently in Section 6 below.

4.1. Bernstein's theorem. A function $\varphi:(0, \infty) \rightarrow \mathbb{R}$ is completely monotone iff

$$
\varphi(t)=\int_{[0, \infty)} e^{-\lambda t} d m(\lambda) \quad(t \in(0, \infty))
$$

for some (unique) Radon measure $m$ on $[0, \infty)$.

Proof. This is a version of a famous theorem of S. Bernstein. See [2, p. 135] for a reduction to the case where $\lim _{t \rightarrow 0} \varphi(t)$ is finite, and, e. g., Mattner [19] for a short proof of this latter case.

4.2. The basic $\mathcal{B}_{k}$ functions. As said before, the letter $\mathcal{B}$, refering to S. Bernstein, has been chosen in view of 4.1. Note, however, that some authors, including Berg, Christensen \& Ressel [2, pp. 114, 141-142], call the members of a subset of $-\mathcal{B}_{1}$ Bernstein functions.

In order to get a useful analogue of Bernstein's theorem 4.1 for functions with some derivative completely monotone, put

$$
\rho_{0, \lambda}(t):=e^{-\lambda t} \quad(\lambda, t \in[0, \infty))
$$

and, for every nonnegative integer $k$,

$$
\rho_{k+1, \lambda}(t):=-\int_{0}^{t} \rho_{k, \lambda}(\tau) d \tau \quad(\lambda, t \in[0, \infty))
$$

Clearly,

$$
\rho_{k, \lambda}(t)=\frac{1}{\lambda^{k}}\left(e^{-\lambda t}-\sum_{l=0}^{k-1} \frac{(-\lambda t)^{l}}{l !}\right) \quad\left(k \in \mathbb{N}_{0}, \lambda, t \in[0, \infty)\right),
$$

the right hand side being defined by continuity at $\lambda=0$ :

$$
\rho_{k, 0}(t)=\frac{(-)^{k} t^{k}}{k !} \quad\left(k \in \mathbb{N}_{0}, t \in[0, \infty)\right) .
$$

Also observe that

$$
(-)^{k} \rho_{k, \lambda}(t) \geq 0 \quad\left(k \in \mathbb{N}_{0}, \lambda, t \in[0, \infty)\right) .
$$


4.3. Representation theorem for $\mathcal{B}_{k}$ functions. Let $k$ be a nonnegative integer. A function $\varphi:(0, \infty) \rightarrow \mathbb{R}$ is $C^{\infty}$ with $(-)^{k} \varphi^{(k)}$ completely monotone, iff, for every $\varepsilon>0$,

$$
\varphi(\varepsilon+t)=\sum_{l=0}^{k-1} \varphi^{(l)}(\varepsilon) \frac{t^{l}}{l !}+\int_{[0, \infty)} \rho_{k, \lambda}(t) e^{-\varepsilon \lambda} d m(\lambda) \quad(t \in[0, \infty))
$$

for some Radon measure $m$ (which is unique, namely the one associated with $(-)^{k} \varphi^{(k)}$ via Berstein's theorem 4.1).

Proof. For $k=0$ this reduces to Bernstein's theorem. So we may proceed by induction and assume that 4.3 is true for some fixed $k \in \mathbb{N}_{0}$.

If $\varphi$ is given with $(-)^{k+1} \varphi^{(k+1)}$ completely monotone, then we may apply the induction hypothesis to $-\varphi^{\prime}$, yielding, for every $\varepsilon>0$,

$$
-\varphi^{\prime}(\tau)=\sum_{l=0}^{k-1} \frac{\left(-\varphi^{\prime}\right)^{(l)}(\varepsilon)}{l !} \tau^{l}+\int_{[0, \infty)} \rho_{k, \lambda}(\tau) e^{-\varepsilon \lambda} d m(\lambda) \quad(\tau \in[0, \infty)) .
$$

Performing $\int_{0}^{t} \ldots d \tau$ on each term in (19), using Fubini, nonnegativity of $(-)^{k} \rho_{k, \lambda}$, and (15), yields (18) with $k$ replaced by $k+1$.

Conversely, if $\varphi$ is a function satisfying (18) with $k$ replaced by $k+1$, a reversion of the above Fubini argument shows, for every $\varepsilon>0$, that $\varphi(\varepsilon+\cdot)$ is absolutely continuous with $-\varphi^{\prime}(\varepsilon+\cdot)$ as in (19). Hence, by the induction hypothesis, $m$ is unique as stated, and $(-)^{k+1} \varphi^{(k+1)}=(-)^{k}\left(-\varphi^{\prime}\right)^{(k)}$ is completely monotone.

4.4. Remark. For $k \geq 1$ it is not possible in general to let $\varepsilon \rightarrow 0$ in (18). For example, if $\varphi(t)=-\log t$, then $-\varphi^{\prime}$ is completely monotone, and we have

$$
\varphi(\varepsilon+t)=-\log \varepsilon+\int_{0}^{\infty}\left(e^{-\lambda t}-1\right) e^{-\varepsilon \lambda} \frac{d \lambda}{\lambda} .
$$

4.5. Behaviour at zero. Let $\varphi:(0, \infty) \rightarrow \mathbb{R}$ be $C^{\infty}$ with $(-)^{k} \varphi^{(k)}$ completely monotone. Then $\lim _{t \rightarrow 0} \varphi(t) \in(-\infty,+\infty]$. If $\lim _{t \rightarrow 0} \varphi(t)=+\infty$, then $\lim _{t \rightarrow 0} \varphi^{\prime}(t)$ $=-\infty$.

Proof. The first claim follows from nonnegativity of $(-)^{n} \varphi^{(n)}$ with, say, $n=$ $\max \{1, k\}$, via the Taylor formula

$$
\varphi(t)=\sum_{l=0}^{n-1} \frac{\varphi^{(l)}(A)}{l !}(t-A)^{l}+\int_{(0, A]} \frac{(s-t)_{+}^{n-1}}{(n-1) !}(-)^{n} \varphi^{(n)}(s) d s \quad(0<t<A) .
$$

The second claim follows from an analogous representation of $-\varphi^{\prime}$ and the fact that $\liminf _{t \rightarrow 0} \varphi^{\prime}(t)=-\infty$.

4.6. Behaviour at infinity. If $k \in \mathbb{N}_{0}, \varphi \in \mathcal{B}_{k}$, and if $m$ is as in (18), then

$$
\lim _{t \rightarrow \infty} \frac{(-)^{k} \varphi(t)}{t^{k}}=\frac{m(\{0\})}{k !} \in[0, \infty) .
$$

If $k \geq 1$ and $\varphi \in \mathcal{B}_{k} \backslash \mathcal{B}_{k-1}$, then

$$
\lim _{t \rightarrow \infty} \frac{(-)^{k} \varphi(t)}{t^{k-1}}=\lim _{t \rightarrow \infty} \frac{(-)^{k} \varphi^{\prime}(t)}{(k-1) t^{k-2}}=\ldots=\lim _{t \rightarrow \infty} \frac{(-)^{k} \varphi^{(k-1)}(t)}{(k-1) !} \in(0,+\infty] .
$$


Proof. By de l'Hospital's rule and (14) with $(-)^{k} \varphi^{(k)}$ in place of $\varphi$,

$$
\lim _{t \rightarrow \infty} \frac{(-)^{k} \varphi(t)}{t^{k}}=\lim _{t \rightarrow \infty} \frac{(-)^{k} \varphi^{(k)}(t)}{k !}=\frac{m(\{0\})}{k !} .
$$

Now assume $k \geq 1$ and $\varphi \in \mathcal{B}_{k} \backslash \mathcal{B}_{k-1}$. By de l'Hospital again, we may assume that $k=1$. Then, applying 4.3 and the isotone convergence theorem, we get

$$
\begin{aligned}
c & :=\lim _{t \rightarrow \infty} \varphi(t)=\lim _{t \rightarrow \infty} \varphi(t+\varepsilon) \\
& =\varphi(\varepsilon)-\infty \cdot m(\{0\})-\int_{(0, \infty)} \frac{e^{-\varepsilon \lambda}}{\lambda} d m(\lambda) \\
& \in[-\infty, \infty)
\end{aligned}
$$

for every $\varepsilon>0$. If we had $c \in[0, \infty)$, then $m(\{0\})=0$ and the above equation for $\varphi(\varepsilon)$ would yield via Bernstein's theorem 4.1 (the easy half) that $\varphi \in \mathcal{B}_{0}$. Since this has been excluded, $c \in[-\infty, 0)$, so (20) is true.

4.7. A decomposition. For every $\varphi \in \mathcal{B}_{k} \backslash \mathcal{B}_{k-1}$ there exists a decomposition

$$
[0, \infty)=[0, A) \cup[A, B) \cup[B, \infty), \quad \varphi=\varphi_{0}+\varphi_{A}+\varphi_{B},
$$

with $0 \leq A<B \leq \infty$, such that

$$
\begin{aligned}
\varphi_{0} & =\varphi \cdot 1_{[0, A)} & & \text { is } \geq 0 \text { and decreasing, } \\
\varphi_{A} & =\varphi \cdot 1_{[A, B)} & & \text { is bounded, } \\
(-)^{k} \varphi_{B} & =(-)^{k} \cdot \varphi \cdot 1_{[B, \infty)} & & \text { is } \geq 0 \text { and increasing }
\end{aligned}
$$

and

$$
|\varphi(\varepsilon+t)-\varphi(t)| \leq C_{1} \cdot \varepsilon \cdot(\varepsilon+t)^{k-1} \quad(t \geq B, \varepsilon \geq 0)
$$

for some constant $C_{1}$. We have $\varphi_{B} \equiv 0$ iff $k=0$.

Further, the function $\psi$ defined by

$$
\psi(t):=(-)^{k} \cdot \varphi_{B}\left(t^{2}\right) \quad(t \in[0, \infty))
$$

is nonnegative, increasing, and satisfies

$$
\psi(s+t) \leq C_{2} \cdot(1+\psi(s)) \cdot(1+\psi(t)) \quad(s, t \in[0, \infty))
$$

for some constant $C_{2}$.

Proof. For $k=0$ take $A=1, B=\infty, C=0$. For $k \geq 1$ use the lemma 4.5 and continuity of $\varphi$ to find an $A \geq 0$ such that $\left.\varphi\right|_{[0, A)}$ and $\left.\varphi\right|_{[A, B)}$ have the required properties for every finite $B>A$. For any $\operatorname{such} B$, we have for some $\xi=\xi(t, \varepsilon) \in$ $(t, t+\varepsilon)$

$$
\left|\frac{\varphi(\varepsilon+t)-\varphi(t)}{(\varepsilon+t)^{k}-t^{k}}\right|=\left|\frac{\varphi^{\prime}(\xi)}{k \cdot \xi^{k-1}}\right| \leq C_{B} \cdot \frac{1}{k} \quad(t \geq B, \varepsilon>0),
$$

since, by the lemma 4.6 , the middle term has a finite limit as $\xi \rightarrow \infty$. By observing that $\left|(\varepsilon+t)^{k}-t^{k}\right| \leq \varepsilon \cdot k \cdot(\varepsilon+t)^{k-1}$, we get (22) with $C_{B}$ in place of $C_{1}$. Use (20) to choose $B$ so large that $(-)^{k} \varphi, \ldots,(-)^{k} \varphi^{(k-1)}$ are strictly positive on $[B, \infty)$. This proves the claims involving (21) and (22) for $k \geq 2$, and $\varphi_{B} \not \equiv 0$ for $k \geq 1$. For $k=1$ we use also the definition of $\mathcal{B}_{1}$ to conclude that $-\varphi^{\prime} \geq 0$.

It remains to prove $(23)$. We claim that $\psi_{B}$ in fact satisfies

$$
\psi_{B}(s+t) \leq 2^{k} \psi_{B}(s)+2^{k} \psi_{B}(t)+C \cdot\left(1+s^{2}\right)^{k-1} \cdot\left(1+t^{2}\right)^{k-1} \quad(s, t \in[0, \infty))
$$

for some constant $C \in[0, \infty)$. 
If $k=0$, then $\psi=0$, so (24) trivially holds for any $C$. So assume $k \geq 1$.

Observe that $(s+t)^{2} \leq 2 s^{2}+2 t^{2}$ and that $(-)^{k} \varphi_{B}$ is increasing. Thus

$$
\psi_{B}(s+t) \leq(-)^{k} \varphi_{B}\left(2 s^{2}+2 t^{2}\right) \quad(s, t \in[0, \infty)) .
$$

Put

$$
\omega(t):=\int_{[B, \infty)}(t-s)_{+}^{k} d \xi(s) \quad(t \in[0, \infty))
$$

where, by concavity of $(-)^{k} \varphi^{(k-1)}$,

$$
d \xi(s):=\frac{(-)^{k}}{(k+1) !} \varphi^{(k+1)}(s) d s
$$

is a signed measure $\leq 0$. Then $\omega$ is subadditive (since it is concave with $\omega(0)=0$ ) and satisfies

$$
\omega(2 t) \leq 2^{k} \omega(t) \quad(t \in[0, \infty))
$$

(since $t \mapsto(t-s)_{+}^{k}$ satisfies for each $s \geq 0$ the reversed inequality). This yields

$$
\omega\left(2 s^{2}+2 t^{2}\right) \leq 2^{k} \omega\left(s^{2}\right)+2^{k} \omega\left(t^{2}\right) \quad(s, t \in[0, \infty)) .
$$

Of course, we have

$$
(-)^{k} \varphi_{B}(t)=p(t)+\omega(t) \quad(t \in[B, \infty))
$$

where

$$
p(t)=\sum_{j=0}^{k} \frac{(-)^{k} \varphi^{(j)}(B)}{j !}(t-B)^{j}
$$

is a polynomial of degree $\leq k$.

If now $2 s^{2}+2 t^{2}<B$, then, via (25), the l. h. s. of (24) is zero, so (24) is trivially true. Otherwise we may apply (25), (27) and (26) to get

$$
\begin{aligned}
\psi_{B}\left((s+t)^{2}\right) \leq & p\left(2 s^{2}+2 t^{2}\right)-2^{k} p\left(s^{2}\right)-2^{k} p\left(t^{2}\right) \\
& +2^{k}\left(p\left(s^{2}\right)+\omega\left(s^{2}\right)+p\left(t^{2}\right)+\omega\left(t^{2}\right)\right) \\
=: & P+\Psi .
\end{aligned}
$$

Now, by (27) and the fact that $\omega$ vanishes on $[0, B]$,

$$
\Psi \leq 2^{k+1} \sup _{0 \leq t \leq B} p(t)+2^{k} \cdot\left(\psi_{B}(s)+\psi_{B}(t)\right) .
$$

Since $P$ is a polynomial in $s^{2}$ and $t^{2}$ of total degree $\leq k$ and with vanishing coefficients of $\left(s^{2}\right)^{k}$ and $\left(t^{2}\right)^{k}$, we finally arrive at $(24)$.

Now (24) implies (23) for some $C_{2}$, using 4.6.

4.8. Remark. Each $\mathcal{B}_{k}$ is obviously a convex cone, and is easily seen to be closed under pointwise convergence. It is known that $\mathcal{B}_{0}$ is closed under multiplication, and that $\varphi \circ \psi \in \mathcal{B}_{0}$ whenever $\varphi \in \mathcal{B}_{0}$ and $\psi \geq 0$ with $-\psi \in \mathcal{B}_{1}$ (see, e.g., [9, p. 441]). These latter properties do not seem to admit interesting generalizations to $\mathcal{B}_{k}$ with larger $k$ : For instance, the product $(-t) \cdot e^{-t}$ and the composition $\exp \left(-t^{2}\right)$ show that, in obvious notation, $\mathcal{B}_{1} \cdot \mathcal{B}_{0} \not \subset \bigcup_{k \geq 0} \pm \mathcal{B}_{k}$ and $\mathcal{B}_{0} \circ \mathcal{B}_{2} \not \subset \bigcup_{k \geq 0} \pm \mathcal{B}_{k}$. 


\section{BiLinear INTEGRABILITy}

5.1. A quasi-Cauchy-Schwarz inequality. Let $d \in \mathbb{N}$ and let $\|\cdot\|_{1}$ and $\|\cdot\|_{2}$ be any two norms on $\mathbb{R}^{d}$. Then there is a constant

$$
C=C\left(d,\|\cdot\|_{1},\|\cdot\|_{2}\right) \in[1, \infty)
$$

such that the inequality

$$
\int_{\mathbb{R}^{d}} \int_{\mathbb{R}^{d}} \psi\left(\|x-y\|_{1}\right) d \mu(x) d \nu(y)
$$

$$
\leq C \cdot \sqrt{\int_{\mathbb{R}^{d}} \int_{\mathbb{R}^{d}} \psi\left(\|x-y\|_{2}\right) d \mu(x) d \mu(y) \cdot \int_{\mathbb{R}^{d}} \int_{\mathbb{R}^{d}} \psi\left(\|x-y\|_{2}\right) d \nu(x) d \nu(y)}
$$

holds for every decreasing function $\psi:[0, \infty) \rightarrow[0, \infty]$ and for every pair $(\mu, \nu)$ of nonnegative Radon measures on $\mathbb{R}^{d}$.

Proof. Choose $A \in(0, \infty)$ with

$$
\|\cdot\|_{2} \leq A\|\cdot\|_{1}
$$

and put

$$
\begin{gathered}
\delta:=\max _{x \in \mathbb{R}^{d}} \min _{m \in \mathbb{Z}^{d}}\|x-m\|_{1}, \\
t:=2 \delta A,
\end{gathered}
$$

and

$$
C:=\max _{x \in \mathbb{R}^{d}} \sum_{m, n \in \mathbb{Z}^{d}} 1\left(\|m-n\|_{1} \leq 2 \delta+t\right) \cdot 1\left(\|x-m\|_{1} \leq \delta\right) .
$$

Then (28) is obviously true. To prove (29), consider for $\mu, \nu \in M_{+}\left(\mathbb{R}^{d}\right)$

$$
I:=\int_{\mathbb{R}^{d}} \int_{\mathbb{R}^{d}} 1\left(\|x-y\|_{1} \leq t\right) d \mu(x) d \nu(y) .
$$

Using the definition of $\delta$ and the inequality $\|m-n\|_{1} \leq\|m-x\|_{1}+\|x-y\|_{1}+\|y-n\|_{1}$, we get

$$
I \leq \sum_{m, n \in \mathbb{Z}^{d},\|m-n\|_{1} \leq 2 \delta+t} \int_{\mathbb{R}^{d}} \int_{\mathbb{R}^{d}} 1\left(\|x-m\|_{1} \leq \delta\right) \cdot 1\left(\|y-n\|_{1} \leq \delta\right) d \mu(x) d \nu(y) .
$$

Now the integrals in the above sum factor by Fubini, so we may apply first the Cauchy-Schwarz inequality, then Fubini again, in order to get

$I \leq \sqrt{\sum_{m, n \in \mathbb{Z}^{d},\|m-n\|_{1} \leq 2 \delta+t} \iint 1\left(\|x-m\|_{1} \leq \delta\right) \cdot 1\left(\|y-m\|_{1} \leq \delta\right) d \mu(x) d \mu(y) \cdot \ldots}$, where the dots ... denote a sum similar to the preceding, with $\nu$ in place of $\mu$. Now use

$$
1\left(\|x-m\|_{1} \leq \delta\right) \cdot 1\left(\|y-m\|_{1} \leq \delta\right) \leq 1\left(\|x-y\|_{2} \leq 2 \delta A\right) \cdot 1\left(\|x-m\|_{1} \leq \delta\right)
$$

and the definition of $C$ to deduce that

$I \leq C \cdot \sqrt{\iint 1\left(\|x-y\|_{2} \leq 2 \delta A\right) d \mu(x) d \mu(y) \cdot \iint 1\left(\|x-y\|_{2} \leq 2 \delta A\right) d \nu(x) d \nu(y)}$. 
This proves (29) for the function

$$
\psi_{t}:=1_{[0, t]}
$$

when $t=2 \delta A$, for arbitrary $\mu$ and $\nu$.

By a change of variables (29) then holds, with the same constant, for all $\psi_{t}$ with $t \in(0, \infty)$. Also, (29) is true for $\psi_{0}=1_{\{0\}}$. (Apply a limit argument for $t \downarrow 0$, or check directly that (29) is in this case true whenever $C \geq 1$, which is the case for our present $C$.)

Now fix $\mu, \nu$ and let $\Psi$ denote the set of all $\psi$ for which (29) is true. By the isotone convergence theorem, $\Psi$ is closed under increasing sequential limits. Also, $\Psi$ is a convex cone: Closedness under addition may be checked by using the implication

$$
\begin{aligned}
u \leq C \cdot \sqrt{v w}, x \leq C \cdot \sqrt{y z} \Rightarrow(u+x)^{2} \leq & C^{2} \cdot(v+y) \cdot(w+z) \\
& (u, v, w, x, y, z \in[0, \infty),
\end{aligned}
$$

which is seen to be true using $2 \cdot \sqrt{v w y z} \leq v z+y w$.

Hence our claim follows on writing an arbitrary $\varphi$ as an increasing sequential limit of positive linear combinations of the functions $\left\{\psi_{t}: t \in[0, \infty)\right\}$.

5.2. A property of increasing, suboperative functions. Let $\psi:[0, \infty) \rightarrow$ $[0, \infty)$ be an increasing function, not identically zero, satisfying for some $C \in[0, \infty)$ the inequalities

$$
\psi(s+t) \leq C \cdot(1+\psi(s)) \cdot(1+\psi(t)) \quad(s, t \in[0, \infty)) .
$$

Let $d \in \mathbb{N}$ and let $\|\cdot\|$ be any norm on $\mathbb{R}^{d}$. Then we have for $\mu, \nu \in M_{+}\left(\mathbb{R}^{d}\right) \backslash\{0\}$ the implications

$$
\begin{aligned}
& \int_{\mathbb{R}^{d}} \int_{\mathbb{R}^{d}} \psi(\|x-y\|) d \mu(x) d \nu(y)<\infty \\
\Longleftrightarrow & \iint \psi(\|x-y\|) d \mu(x) d \mu(y)+\iint \psi(\|x-y\|) d \nu(x) d \nu(y)<\infty \\
\Longleftrightarrow & \int_{\mathbb{R}^{d}} \psi(\|x\|) d \mu(x)+\int_{\mathbb{R}^{d}} \psi(\|x\|) d \nu(x)<\infty \\
\Longrightarrow & \mu, \nu \text { bounded. }
\end{aligned}
$$

Proof. As to the name of this proposition: The condition (30) may alternatively be stated as follows: The function $\Psi=C+\psi$ is, for some choice of $C$, a suboperative function from the semigroup $([0, \infty),+)$ into the ordered semigroup $([1, \infty), \cdot, \leq)$, i.e.,

$$
\Psi(s+t) \leq \Psi(s) \cdot \Psi(t) \quad(s, t \in[0, \infty)) .
$$

In other words: $\log \Psi$ is required to be subadditive. See [13, Chapter 13] for information on such functions.

Coming now to the proof proper, assume that (31), (32) or (33) is true. Then, by Fubini or trivially,

$$
\int_{\mathbb{R}^{d}} \psi\left(\left\|x-x_{1}\right\|\right) d \mu(x)+\int_{\mathbb{R}^{d}} \psi\left(\left\|x-x_{2}\right\|\right) d \nu(x)<\infty
$$

for some $x_{1}, x_{2} \in \mathbb{R}^{d}$. Now $\psi(t) \geq \delta \cdot 1\left(t \geq t_{0}\right)$ for some $\delta>0$ and some $t_{0} \in[0, \infty)$. Since (35) is trivially true when $\psi(t)$ is replaced by $1\left(t \leq t_{0}\right)$, the measures $\mu, \nu$ being finite on compact sets, (34) follows. 
Using $\|x-y\| \leq\|x\|+\|y\|$ and the assumption that $\psi$ is increasing, we have

$$
\psi(\|x-y\|) \leq C \cdot(1+\psi(\|x\|)) \cdot(1+\psi(\|y\|)) \quad\left(x, y \in \mathbb{R}^{d}\right) .
$$

Assume (33). Then, by (34), (36) obviously implies (31) and (32).

Now assume (31) or (32). Then (35) is true. By applying (36), with $x$ replaced by $x-x_{1}$ and $y$ replaced by $x_{1}$, and using (34), we get the finiteness of the first integral in (33). Similarly, we get finiteness of the second. Thus (33) is true.

5.3. Some vector spaces of bilinearly integrable measures. Let $d \in \mathbb{N}$ and let $\|\cdot\|$ be any norm on $\mathbb{R}^{d}$. Let $\psi:[0, \infty) \rightarrow[0, \infty]$ be

i) decreasing, or

ii) finite valued, increasing, and satisfying (30) for some $C \in[0, \infty)$.

Then

$$
M_{\psi}:=\left\{\sigma \in M\left(\mathbb{R}^{d}\right): \int_{\mathbb{R}^{d}} \int_{\mathbb{R}^{d}} \psi(\|x-y\|) d|\sigma|(x) d|\sigma|(y)<\infty\right\}
$$

is a vector subspace of $M\left(\mathbb{R}^{d}\right)$, and the map

$$
(\sigma, \tau) \mapsto \iint \psi(\|x-y\|) d \sigma(x) d \tau(y) \quad\left(\sigma, \tau \in M_{\psi}\right)
$$

is a well-defined real-valued bilinear form on $M_{\psi}$.

Under the assumption i), $M_{\psi} \neq\{0\}$ iff $M_{\psi}$ contains $L^{1}\left(\mathbb{R}^{d}\right) \cap L^{2}\left(\mathbb{R}^{d}\right)$ iff

$$
\int_{0}^{1} \psi(r) r^{d-1} d r<\infty
$$

Under the assumption ii), $M_{\psi}$ always contains the space of all signed measures with compact support.

Proof. In both cases, we have the implication

$$
\mu, \nu \in M_{\psi}, \mu, \nu \geq 0 \Longrightarrow \iint \psi(\|x-y\|) d \mu(x) \nu(y)<\infty,
$$

by 5.1 and 5.2 . By the inequality

$$
|\sigma+\tau| \otimes|\sigma+\tau| \leq|\sigma| \otimes|\sigma|+|\tau| \otimes|\tau|+|\sigma| \otimes|\tau|+|\tau| \otimes|\sigma|
$$

it hence follows that $M_{\psi}$ is closed under addition, thus a vector space. The statement concerning the bilinear form also follows easily from (39).

The last statement, refering to $M_{\psi}$ under assumption ii), is trivial.

It remains to prove the stated equivalence involving (38). So assume i). By (29) with $\mu=\nu=|\sigma|$, we may assume that $\|\cdot\|$ is the euclidean norm.

Assume (38) and let $f \in L^{1}\left(\mathbb{R}^{d}\right) \cap L^{2}\left(\mathbb{R}^{d}\right)$ with $f \geq 0$. Then $g=f * \check{f}$, the convolution of $f$ with its reflection, belongs to $L^{1}\left(\mathbb{R}^{d}\right)$ and is bounded. Writing

$$
\iint \psi(\|x-y\|) f(x) d x f(y) d y=\int \psi(\|x\|) g(x) d x=\int_{\|x\| \leq 1}+\int_{\|x\|>1},
$$

applying boundedness of $g$, polar coordinates, and (38) to the first integral on the r.h.s., and boundedness of $\psi(\|x\|)$ away from the origin and $g \in L^{1}\left(\mathbb{R}^{d}\right)$ to the second, we see that the l.h.s. above is finite. Thus $f \in M_{\psi}$. 
Now assume that (38) is false and let $\mu \in M_{+}\left(\mathbb{R}^{d}\right) \backslash\{0\}$. Let $B(x, r)$ denote the closed ball with center $x$ and radius $r$, and let $f(x)$ denote the lower symmetric density of $\mu$ with respect to Lebesgue measure $\lambda^{d}$, i. e.,

$$
f(x):=\liminf _{r \rightarrow 0} \frac{\mu(B(x, r))}{\lambda^{d}(B(x, r))} \quad\left(x \in \mathbb{R}^{d}\right) .
$$

Then

$$
A:=\left\{x \in \mathbb{R}^{d}: f(x)>0\right\}
$$

satisfies $\mu\left(A^{c}\right)=0$, by Rudin [24, Theorem 8.6] and a theorem of Besicovitch [3] as stated in [24, Notes to Sec. 8.10,8.11]. Hence $\mu(A)>0$.

Fix $x \in A$. Choose $r_{0}>0$ such that

$$
\mu(B(x, r)) \geq \frac{1}{2} f(x) \cdot \lambda^{d}(B(x, r)) \quad\left(r \in\left[0, r_{0}\right]\right) .
$$

This may be rewritten as

$$
\int_{\mathbb{R}^{d}} 1(\|y-x\| \leq r) d \mu(y) \geq \frac{1}{2} f(x) \cdot \int_{\mathbb{R}^{d}} 1(\|y-x\| \leq r) d \lambda^{d}(y) \quad\left(r \in\left[0, r_{0}\right]\right) .
$$

Put $\psi_{0}:=\psi \cdot 1_{\left[0, r_{0}\right]}$. Then, using an approximation argument as in the proof of 5.1, polar coordinates, and the negation of (38), we get

$$
\begin{aligned}
\int \psi(\|y-x\|) d \mu(y) & \geq \int \psi_{0}(\|y-x\|) d \mu(y) \\
& \geq \frac{1}{2} f(x) \cdot \int \psi_{0}(\|y-x\|) d \lambda^{d}(y) \\
& =\frac{1}{2} f(x) \cdot \int \psi(r) \omega_{d} r^{d-1} d r \\
& =\infty .
\end{aligned}
$$

Hence

$$
\begin{aligned}
\iint \psi(\|y-x\|) d \mu(y) d \mu(x) & \geq \int_{A} \int_{\mathbb{R}^{d}} \psi(\|y-x\|) d \mu(y) d \mu(x) \\
& =\int_{A} \infty d \mu(x) \\
& =\infty
\end{aligned}
$$

and so $\mu \notin M_{\psi}$. It follows that $M_{\psi}=\{0\}$.

Trivially, $L^{1}\left(\mathbb{R}^{d}\right) \cap L^{2}\left(\mathbb{R}^{d}\right) \subset M_{\psi}$ implies that $M_{\psi} \neq\{0\}$.

5.4. Remark on Besicovitch' covering theorem. The serious reader will already have noticed that there is an inaccuracy in the proof of the covering theorem $[3$, Theorem], the theorem that forms the basis of the differentiation theorem cited and used in the above proof of 5.3. Namely, the claim that $G$ is covered in [3, p. 106 , lines 8,9$]$ is not necessarily true if $G$ is unbounded. Also the construction of the sequence $\left(c_{n}: n \in \mathbb{N}\right)$ is not clear when $\Gamma$ contains arbitrarily large circles. These problems may, however, easily be circumvented via replacing the original covering $\Gamma$ by $\tilde{\Gamma}:=\left\{c(O, r) \in \Gamma:|r| \leq(1+|O|)^{-1}\right\}$. Further, observe that [3, Lemma 2.1] should be followed by a remark analogous to that following [3, Lemma 2], and that the proof of [3, Theorem] should refer to the remark suggested. 
5.5. Bilinear integrability need not define a vector space. The vector space property of $M_{\psi}$ as in (37) does not come for free. As an easy example, consider

$$
\psi(t):=\left|\sin \frac{\pi}{2} t\right| \quad(t \in[0, \infty))
$$

and define $M_{\psi}$ as above, say with $d=1$. Put $\mu=\sum_{k=1}^{\infty} \delta_{2 k}$ and $\nu=\sum_{k=1}^{\infty} \delta_{2 k-1}$, $\delta_{x}$ denoting the Dirac measure located at $x$. Then both $\mu$ and $\nu$ belong to $M_{\psi}$, but their sum $\mu+\nu=\sum_{k=1}^{\infty} \delta_{k}$ does not. So $M_{\psi}$ is no vector space, and the implication $(32) \Longrightarrow(31)$ is false, in spite of $\psi$ satisfying (30).

\section{The REMAining PRoOfS}

In this section, $\|\cdot\|$ always denotes the euclidean norm, i.e., $\|x\|=\sqrt{x \cdot x}$, with "." denoting the usual inner product.

6.1. A moment problem. If $\sigma$ is a signed Radon measure on $\mathbb{R}^{d}$, and $t \in(0, \infty)$ with

$$
\iint e^{t x \cdot y} d|\sigma|(x) d|\sigma|(y)<\infty
$$

then

$$
\begin{aligned}
\iint e^{t x \cdot y} d \sigma(x) d \sigma(y) & =\sum_{l=0}^{\infty} \frac{t^{l}}{l !} \iint(x \cdot y)^{l} d \sigma(x) d \sigma(y) \\
& =\sum_{l=0}^{\infty} \frac{t^{l}}{l !} \sum_{|\alpha|=l}\left(\begin{array}{l}
l \\
\alpha
\end{array}\right) \cdot\left(\int x^{\alpha} d \sigma(x)\right)^{2},
\end{aligned}
$$

and this equals zero iff $\sigma=0$.

Proof. By a change of variables, we may assume that $t=1$. In order to justify the obvious applications of Fubini's theorem leading to the claimed equalities, in particular showing the existence of all moments of $\sigma$, it suffices to show that

$$
I:=\iint \exp \left(\left|x_{1} y_{1}\right|+\ldots+\left|x_{d} y_{d}\right|\right) d|\sigma|(x) d|\sigma|(y)<\infty .
$$

Suppose first that $\sigma$ has compact support. Then

$$
\begin{aligned}
I_{l}(\varepsilon) & :=\iint\left(\varepsilon_{1} x_{1} y_{1}+\ldots+\varepsilon_{d} x_{d} y_{d}\right)^{l} d|\sigma|(x) d|\sigma|(y) \\
& =\sum_{|\alpha|=l}\left(\begin{array}{l}
l \\
\alpha
\end{array}\right) \varepsilon^{\alpha}\left(\int x^{\alpha} d|\sigma|(x)\right)^{2} \\
& \leq I_{l}(1, \ldots, 1) \quad\left(\varepsilon \in\{-1,1\}^{d}\right) .
\end{aligned}
$$

Since $\exp \left(\left|x_{1} y_{1}\right|+\ldots+\left|x_{d} y_{d}\right|\right) \leq \sum_{\varepsilon} \exp \left(\varepsilon_{1} x_{1} y_{1}+\ldots+\varepsilon_{d} x_{d} y_{d}\right)$, it follows that

$$
I \leq 2^{d} \iint e^{x \cdot y} d|\sigma|(x) d|\sigma|(y) .
$$

By monotone convergence, this inequality remains true for arbitrary $\sigma$. Thus $I<\infty$ by assumption (40).

Now $\iint e^{t x \cdot y} d \sigma(x) d \sigma(y)=0$ iff all moments of $\sigma$ vanish. So it remains to be shown that the latter implies $\sigma=0$. To this end, it suffices to prove that

$$
M(y):=\int e^{x \cdot y} d|\sigma|(x)
$$


is finite in a neighbourhood of the origin: then, via analyticity, the Fourier transform of $\sigma$ would be zero.

So assume that 0 is not an interior point of

$$
C:=\left\{y \in \mathbb{R}^{d}: M(y)<\infty\right\} .
$$

Then, by convexity of $C$, there is an $l \in \mathbb{R}^{d} \backslash\{0\}$ such that

$$
l \cdot y \geq 0 \quad(y \in C)
$$

In particular, $-l \notin C$. On the other hand, since $\int M(y) d|\sigma|(y)<\infty, C$ contains a measurable support $S$ of $|\sigma|$ (i.e., a Borel set $S$ satisfying $|\sigma|\left(\mathbb{R}^{d} \backslash S\right)=0$ ). Thus $l \cdot y \geq 0$ for $|\sigma|$-almost every $y$; hence

$$
\int e^{-l \cdot y} d|\sigma|(y) \leq|\sigma|\left(\mathbb{R}^{d}\right)
$$

and this is finite since we already know that $\int x^{0} d \sigma(x)$ is finite. Thus $-l \in C$, a contradiction.

6.2. The basic inequalities. Let $k$ be a nonnegative integer and let $\sigma$ be a signed measure on $\mathbb{R}^{d}$ such that

$$
\int_{\mathbb{R}^{d}} x^{\alpha} d \sigma(x)=0 \quad\left(\alpha \in \mathbb{N}_{0}^{d},|\alpha| \leq k-1\right) .
$$

Then each of the following equalities holds whenever its left hand side is finite:

$$
\begin{aligned}
& I\left(\rho_{k, 0} ; \sigma, \sigma\right)=\sum_{l=0}^{\left\lfloor\frac{k}{2}\right\rfloor} \frac{2^{k-2 l}}{(l !)^{2}(k-2 l) !} \sum_{|\alpha|=k-2 l}\left(\begin{array}{c}
k-2 l \\
\alpha
\end{array}\right)\left(\int_{\mathbb{R}^{d}} x^{\alpha}\|x\|^{2 l} d \sigma(x)\right)^{2}, \\
& I\left(\rho_{k, \lambda} ; \sigma, \sigma\right)=\frac{1}{\lambda^{k}} \sum_{l=0}^{\infty} \frac{2^{l} \lambda^{l}}{l !} \sum_{|\alpha|=l}\left(\begin{array}{c}
l \\
\alpha
\end{array}\right)\left(\int_{\mathbb{R}^{d}} x^{\alpha} e^{-\lambda\|x\|^{2}} d \sigma(x)\right)^{2} \quad(\lambda>0) .
\end{aligned}
$$

We have $I\left(\rho_{k, 0} ; \sigma, \sigma\right)=0$ iff $\int\|x\|^{2 k} d|\sigma|(x)<\infty$ and (41) holds with $k-1$ replaced by $k$, and we have $I\left(\rho_{k, \lambda} ; \sigma, \sigma\right)=0$ for some $\lambda>0$ iff $\sigma=0$.

Proof. To prove (42), note that finiteness of the l. h. s. is equivalent to $\int\|x\|^{2 k} d|\sigma|(x)$ $<\infty$. So we may proceed as in $[20$, p. 16] (there $k$ occurs four times as a misprint for $k-l$ ) by writing

$$
\|x-y\|^{2 k}=\sum_{l \in \mathbb{N}_{0}^{3},|l|=k}\left(\begin{array}{l}
k \\
l
\end{array}\right)(-2)^{l_{3}} \sum_{|\alpha|=l_{3}}\left(\begin{array}{l}
l_{3} \\
\alpha
\end{array}\right) x^{\alpha}\|x\|^{2 l_{1}} y^{\alpha}\|y\|^{2 l_{2}},
$$

then observing that, by (41), only terms with $l_{1}=l_{2}$ contribute to $\iint \ldots d \sigma d \sigma$. Hence

$$
\begin{aligned}
\iint & \|x-y\|^{2 k} d \sigma(x) \sigma(y) \\
= & \sum_{l=0}^{\left\lfloor\frac{k}{2}\right\rfloor} \frac{(-)^{k} k ! 2^{k-2 l}}{(l !)^{2}(k-2 l) !} \sum_{|\alpha|=k-2 l}\left(\begin{array}{c}
k-2 l \\
\alpha
\end{array}\right)\left(\int_{\mathbb{R}^{d}} x^{\alpha}\|x\|^{2 l} d \sigma(x)\right)^{2},
\end{aligned}
$$

and thus (42). From (42), the statement concerning the equality $I\left(\rho_{k, 0} ; \sigma, \sigma\right)=0$ easily follows. 
Now let $\lambda>0$ be such that $I\left(\rho_{k, \lambda} ; \sigma, \sigma\right)$ is finite. Apply (41) to yield, by what has just been proved, $\iint\|x-y\|^{2 j} d \sigma(x) d \sigma(y)=0(j=0, \ldots, k-1)$. Thus

$$
\begin{aligned}
I\left(\rho_{k, \lambda} ; \sigma, \sigma\right) & =\frac{1}{\lambda^{k}} \iint e^{-\lambda\|x-y\|^{2}} d \sigma(x) d \sigma(y) \\
& =\frac{1}{\lambda^{k}} \iint e^{2 \lambda x \cdot y} e^{-\lambda\|x\|^{2}} d \sigma(x) e^{-\lambda\|y\|^{2}} d \sigma(y) .
\end{aligned}
$$

Now an application of the lemma 6.1 (with $2 \lambda$ in place of $t$ and $\exp \left(-\lambda\|\cdot\|^{2}\right) \sigma$ in place of $\sigma$ ) yields (43), as well as the statement concerning $I\left(\rho_{k, \lambda} ; \sigma, \sigma\right)=0$.

\subsection{Proof of the Theorem.}

Step 1. There is no loss in generality in assuming that $\varphi \in \mathcal{B}_{k} \backslash \mathcal{B}_{k-1}$ in the theorem.

Proof. Assume that the theorem is true under the more stringent assumption " $\varphi \in$ $\mathcal{B}_{k} \backslash \mathcal{B}_{k-1}$ " instead of " $\varphi \in \mathcal{B}_{k}$ ".

Let $\varphi \in \mathcal{B}_{k}$ and let $\mu, \nu$ satisfy (4). Then $\varphi \in \mathcal{B}_{j} \backslash \mathcal{B}_{j-1}$ for some $j \in\{0, \ldots, k\}$, and (4) is true also when $j$ replaces $k$. Hence the conclusion of the theorem, with $j$ replacing $k$, follows.

This is easily seen to imply that the conclusion of the theorem is also true as it stands, i.e., with $k$ not replaced. The only part in checking this which might be nonobvious concerns the "if" claim in the discussion of equality. But if $\varphi \in \mathcal{B}_{k}$ with $(-)^{k} \varphi^{(k)}=0$ and $k>j$, then $\varphi$ is a polymial of degree $\leq j$, using (10), and thus $(-)^{j} \varphi^{(j)}$ is constant, and $\int x^{\alpha} d \mu(x)=\int x^{\alpha} d \nu(x) \neq \pm \infty(|\alpha|=j)$ by (4). Hence we may assume that $(-)^{k} \varphi^{(k)}$ is a nonzero constant and $\int x^{\alpha} d \mu(x)=\int x^{\alpha} d \nu(x) \neq$ $\pm \infty$ also for $|\alpha|=k$. But then (10) implies $\varphi \in \mathcal{B}_{k} \backslash \mathcal{B}_{k-1}$.

Assumption and notation. For the rest of this proof, we assume $\varphi \in \mathcal{B}_{k} \backslash \mathcal{B}_{k-1}$. Let

$$
\varphi=\varphi_{0}+\varphi_{A}+\varphi_{B}
$$

be a decomposition as in 4.7. Let $m$ denote the measure associated to $\varphi$ via the representation theorem 4.3. For $\varepsilon>0$ put

$$
\varphi_{\varepsilon}:=\varphi(\varepsilon+\cdot) \text {. }
$$

Let $\mu, \nu \in M_{+}\left(\mathbb{R}^{d}\right)$.

Step 2. Without assuming (4), but assuming in case $k \geq 1$ and $(-)^{k} \cdot(I(\varphi ; \mu, \mu)+$ $I(\varphi ; \nu, \nu))=\infty$ the following consequences of $(4)$ :

$$
\begin{gathered}
\mu, \nu \text { bounded, } \\
\mu=0 \Longleftrightarrow \nu=0,
\end{gathered}
$$

we have:

i) If the r.h.s. of (5) exists, then so does the l.h.s.

ii) If the r.h.s. of (5) is finite, then so is the l.h.s.

iii) If the r.h.s. of (5) exists, and if one of the three integrals $I(\varphi ; \mu, \nu), I(\varphi ; \mu, \mu)$, $I(\varphi ; \nu, \nu)$ belongs to $\{-\infty, \infty\}$, then (5) is true.

Thus there is no loss in generality in assuming that the three integrals in (5) are finite. 
Proof. Obviously, (45) and (46) follow from (4) if $k \geq 1$. The "Thus" claim is obvious as well.

Assume first that $k=0$. Then $\varphi \geq 0$, and all three integrals exist and belong to $[0, \infty]$. Hence i) is trivially true.

If the r.h.s. of (5) is $\infty$, then (5) is trivially true. If the r.h.s. of (5) is finite, then we may apply the quasi-Cauchy-Schwarz inequality 5.1, to the function $\psi(t)=$ $\psi\left(t^{2}\right)$ and to the norms $\|\cdot\|_{1}=\|\cdot\|_{2}=\|\cdot\|$, in order to see that $I(\varphi ; \mu, \nu)$ is finite as well. Hence ii) and iii) are true.

Assume now that $k \geq 2$ is even. Referring to the decomposition (44), the three integrals $I\left(\varphi_{0} ; \ldots\right)$ and the three integrals $I\left(\varphi_{B} ; \ldots\right)$ exist and belong to $[0, \infty]$.

The r.h.s. of (5) can never be $-\infty$ : If it were, then we would have $I\left(\varphi_{A} ; \mu, \mu\right)+$ $I\left(\varphi_{A} ; \nu, \nu\right)=-\infty$, hence, say, $\mu$ unbounded, so that 5.2 could be applied to the function $\psi(t)=\varphi_{B}\left(t^{2}\right)$ and to the pair of measures $(\mu, \mu)$, in order to deduce $I\left(\varphi_{B} ; \mu, \mu\right)=\infty$, yielding nonexistence of the r.h.s. of (5).

Assume that the r.h.s. of (5) is finite. Then 5.1, again applied to $\psi(t)=\varphi_{0}\left(t^{2}\right)$ and the euclidean norm, yields finiteness of $I\left(\varphi_{0} ; \mu, \nu\right)$. The integrals $I\left(\varphi_{A} ; \mu, \nu\right)$ and $I\left(\varphi_{B} ; \mu, \nu\right)$ are finite as well: This is trivial if $\mu=0$ or $\nu=0$, and otherwise we may apply 5.2, again to the function $\psi(t)=\varphi_{B}(t)$, but now to the measures $\mu$, $\nu$. Taken together, we have finiteness of the l.h.s. of (5). Thus ii) is true.

If the r.h.s. of (5) is $\infty$, then we use (45) to deduce finiteness of $I\left(\varphi_{A} ; \mu, \nu\right)$, and thus existence of the l.h.s. of (5). Hence i) and iii) are true.

Assume finally that $k \geq 1$ is odd. Again, the integrals $I\left(\varphi_{0} ; \ldots\right)$ and $I\left(\varphi_{B} ; \ldots\right)$ exist, and the former three belong to $[0, \infty]$, but now the latter three belong to $[-\infty, 0]$.

If the r.h.s. of (5) is finite, then we see as in the case $k \geq 2$ even, but replacing $\varphi_{B}\left(t^{2}\right)$ by $-\varphi_{B}\left(t^{2}\right)$, that the l.h.s. of (5) is finite as well. Hence ii) is true.

If the r.h.s. of $(5)$ is $+\infty$, then $I\left(\varphi_{B} ; \mu, \mu\right)+I\left(\varphi_{B} ; \nu, \nu\right)$ must be finite. It follows that $I\left(\varphi_{B} ; \mu, \nu\right)$ and $I\left(\varphi_{A}, \mu, \nu\right)$ are finite: This is trivial if $\mu=0$ or $\nu=0$, and follows using 5.2 otherwise. Hence the l.h.s. of (5) exists and (5) is true.

If the r.h.s. of (5) is $-\infty$, then we use (45) to conclude that the integrals $I\left(\varphi_{A} ; \ldots\right)$ are finite. Thus $I\left(\varphi_{B} ; \mu, \mu\right)+I\left(\varphi_{B} ; \nu, \nu\right)=-\infty$. Using (46) and (5.2), we get $I\left(\varphi_{B} ; \mu, \nu\right)=-\infty$. Thus $I(\varphi: \mu, \nu)=-\infty$ and (5) is true. Thus i) and ii) are true.

Step 3. If $k \geq 1$ and $\mu \otimes \nu \neq 0$, then the following implications are valid:

$$
\int_{\mathbb{R}^{d}}|x|^{2 k} d(\mu+\nu)(x)<\infty \Rightarrow I\left(\psi_{B} ; \mu, \nu\right)<\infty \Rightarrow \int_{\mathbb{R}^{d}}|x|^{2(k-1)} d(\mu+\nu)(x)<\infty .
$$

Proof. By the lemma 4.6 we have

$$
c_{1} \cdot t^{k-1} \leq \psi_{B}(t) \leq C_{2} \cdot t^{k} \quad(t \in[0, \infty))
$$

for some constants $c_{1}, C_{2} \in(0, \infty)$. Hence (47) follows by applying 5.2 once to $\psi(t)=t^{2 k}$ and once to $\psi(t)=t^{2(k-1)}$.

Further assumption. For the rest of this proof, we assume that the three integrals in (5) are finite.

Step 4. $I\left(\varphi_{\varepsilon} ; \mu, \nu\right)$ is finite for every $\varepsilon>0$, and

$$
I(\varphi ; \mu, \nu)=\lim _{\varepsilon \rightarrow 0} I\left(\varphi_{\varepsilon} ; \mu, \nu\right) .
$$


Proof. If $k=0$, then $\varphi$ is $\geq 0$ and antitone, so that the claim follows from the isotone convergence theorem.

So assume that $k \geq 1$. Then $\mu \otimes \nu$ is bounded, by step 3 . Referring to the decomposition (44), we write

$$
\varphi_{\varepsilon}=\left(\varphi_{0}\right)_{\varepsilon}+\left(\varphi_{A}\right)_{\varepsilon}+\left(\varphi_{B}\right)_{\varepsilon}
$$

and check (48) for each of three terms on the r.h.s. of (49) separately. For $\left(\varphi_{0}\right)_{\varepsilon}$ we use antitonicity as in the above argument in the case $k=0$. For $\left(\varphi_{A}\right)_{\varepsilon}$ we may use the dominated convergence theorem, since $\mu \otimes \nu$ and $\varphi_{A}$ are bounded. For $\left(\varphi_{B}\right)_{\varepsilon}$, finally, we observe that (23) yields

$$
\left|\left(\varphi_{B}\right)_{\varepsilon}\left(t^{2}\right)\right| \leq \psi(\varepsilon+t) \leq C_{2} \cdot(1+\psi(\varepsilon)) \cdot\left(1+\left|\varphi_{B}\left(t^{2}\right)\right|\right),
$$

so that the dominated convergence theorem is applicable again.

Step 5. If (4) holds, then

$$
I\left(\rho_{k, \lambda} ; \sigma, \sigma\right) \in[0, \infty)
$$

for $m$-almost every $\lambda \in[0, \infty)$, and

$$
I(\varphi ; \sigma, \sigma)=\int_{[0, \infty)} I\left(\rho_{k, \lambda} ; \sigma, \sigma\right) d m(\lambda) .
$$

Proof. First fix $\varepsilon>0$. By step 4,

$$
I\left(\left|\varphi_{\varepsilon}\right| ;|\sigma|,|\sigma|\right)<\infty .
$$

By the representation theorem 4.3,

$$
\varphi_{\varepsilon}(t)=p_{\varepsilon}(t)+\int_{[0, \infty)} \rho_{k, \lambda}(t) e^{-\varepsilon \lambda} d m(\lambda) \quad(t \geq 0),
$$

where $p_{\varepsilon}$ is a polynomial of degree at most $k-1$ (if $k=0$, this means that $p_{\varepsilon}$ is the zero polynomial).

We claim that

$$
I\left(p_{\varepsilon} ; \sigma, \sigma\right)=0 .
$$

This is trivial for $k=0$. If $k \geq 1$, then step 3 , applied to $(\mu, \nu)=(|\sigma|,|\sigma|)$, yields

$$
I\left(|\cdot|^{k-1} ;|\sigma|,|\sigma|\right)<\infty .
$$

Now (4) and the lemma 6.2 with $\lambda=0$ and $j=0, \ldots, k-1$ in place of $k$ yield (54).

Together with (52) and (53), this yields

$$
I\left(\varphi_{\varepsilon} ; \sigma, \sigma\right)=\int_{\mathbb{R}^{d}} \int_{\mathbb{R}^{d}}\left[\int_{[0, \infty)} \rho_{k, \lambda}\left(\|x-y\|^{2}\right) e^{-\varepsilon \lambda} d m(\lambda)\right] d \sigma(x) d \sigma(y) \in \mathbb{R} .
$$

In view of (17), we may use Fubini to conclude that

$$
I\left(\left|\rho_{k, \lambda}\right| ;|\sigma|,|\sigma|\right)<\infty
$$

for $m$-almost every $\lambda \in[0, \infty)$, and that

$$
I\left(\varphi_{\varepsilon} ; \sigma, \sigma\right)=\int_{[0, \infty)} I\left(\rho_{k, \lambda} ; \sigma, \sigma\right) e^{-\varepsilon \lambda} d m(\lambda) .
$$

By 6.2 we have (50) for every $\lambda$ satisfying (56).

Finally, (51) follows from (57) by letting $\varepsilon \downarrow 0$, using the isotone convergence theorem. 
Step 6. If (4) holds, then (5) is true, with equality occurring exactly under the stated conditions.

Proof. Step 5 yields

$$
I(\varphi ; \sigma, \sigma) \geq 0,
$$

which is equivalent to (5). Further, we have equality in (5) iff we have equality in (58) iff

$$
I\left(\rho_{k, \lambda} ; \sigma, \sigma\right)=0[m]
$$

(to be read as: The stated equality is true for $m$-almost every $\lambda \in[0, \infty)$.).

If $m=0$, then we have equality in (5) and, by $(18),(-)^{k} \varphi^{(k)}=0$.

Let $m((0, \infty))>0$. Then, as may be checked using the uniqueness statement in $4.3,(-)^{k} \varphi^{(k)}$ is not constant. Thus the only possible one of the three stated conditions for equality is $\mu=\nu$, and this condition is, by 6.2 , indeed equivalent to (59).

Finally let $m((0, \infty))=0$ but $m(\{0\})>0$. Then, by $(18),(-)^{k} \varphi^{(k)}$ is constant, and (59) reads

$$
I\left(\rho_{k, 0} ; \sigma, \sigma\right)=0 .
$$

By 6.2 this occurs iff the third of the stated conditions for equality is true, which in the present case, is implied by either of the other two conditions.

This concludes the proof of Theorem 2.2.

6.4. Proof of Remark 2.3 b). Let $k=1, \varphi(t)=\frac{1}{2} \log \frac{1}{t}, d=1$. Put

$$
h(x):=\frac{1}{|x| \cdot(1+|\log | x||)^{2}} \quad(x \in \mathbb{R}) .
$$

Since $h \in L^{1}(\mathbb{R})$, we may by [23] write $h$ as a convolution

$$
h=h_{1} * h_{2}
$$

for some $h_{1}, h_{2} \in L^{1}(\mathbb{R})$. Put

$$
f:=\left|h_{1}\right|+\left|\check{h}_{2}\right|
$$

where $\check{h}_{2}(x):=h_{2}(-x)$. Since $f$ and $\log _{-}|\cdot|$ belong to $L^{1}(\mathbb{R})$, we may choose a $z \in \mathbb{R}$ such that $\left(\log _{-}|\cdot| * f * \check{f}\right)(z)<\infty$, which may be written as

$$
\iint \log _{-}|x-y| f(x) g(y) d x d y<\infty
$$

with

$$
g(x):=f(x+z) \quad(x \in \mathbb{R}) .
$$

Let $\mu, \nu$ be the measures with Lebesgue densities $f, g$. Then

$$
I\left(\varphi_{+} ; \nu, \nu\right)=I\left(\varphi_{+} ; \mu, \mu\right)=\int_{\mathbb{R}} \log _{-} \cdot(f * \check{f}) d x \geq \int_{\mathbb{R}} \log _{-} \cdot h d x=\infty
$$

and, similarly,

$$
I\left(\varphi_{-} ; \nu, \nu\right)=I\left(\varphi_{-} ; \mu, \mu\right)=\infty
$$

Hence, applying 5.2 to $\varphi(t)=\log _{+} t$,

$$
I\left(\varphi_{-} ; \mu, \nu\right)=\infty \text {. }
$$

Finally, (60) means

$$
I\left(\varphi_{+} ; \mu, \nu\right)<\infty
$$


6.5. Proof of Corollary 2.4. We may assume that $I(\varphi ; \sigma, \sigma) \in[-\infty, \infty)$, since otherwise the claim is trivial. By (3) with $\sigma=\tau$, this means that

$$
I\left(\varphi_{+} ; \sigma_{+}, \sigma_{+}\right), I\left(\varphi_{+} ; \sigma_{-}, \sigma_{-}\right), I\left(\varphi_{-} ; \sigma_{+}, \sigma_{-}\right) \in[0, \infty) .
$$

Put $\mu=\sigma_{+}$and $\nu=\sigma_{-}$. Then (4) holds and both sides of (5) are finite, so that Theorem 2.2 yields

$$
-\infty<2 I\left(\varphi ; \sigma_{+}, \sigma_{-}\right) \leq I\left(\varphi ; \sigma_{+}, \sigma_{+}\right)+I\left(\varphi ; \sigma_{-}, \sigma_{-}\right)<\infty .
$$

Now by the theorem again, equality above could only occur if $\sigma_{+}=\sigma_{-}$, which is impossible since $\sigma \neq 0$. Thus

$$
I(\varphi ; \sigma, \sigma)=I\left(\varphi ; \sigma_{+}, \sigma_{+}\right)+I\left(\varphi ; \sigma_{-}, \sigma_{-}\right)-2 I\left(\varphi ; \sigma_{+}, \sigma_{-}\right)>0 .
$$

6.6. Proof of Corollary 2.5. If $k=0$, then $M^{\varphi}\left(\mathbb{R}^{d}\right)$ is a vector space and (9) is a well-defined real-valued bilinear form, by 5.3 applied to the decreasing function $\psi(t)=\varphi\left(t^{2}\right)$.

If $k \geq 1$ and $\sigma \in M^{\varphi}\left(\mathbb{R}^{d}\right)$, then $I\left((-)^{k} \varphi_{B} ;|\sigma|,|\sigma|\right)<\infty$, so that step 3 of the proof of Theorem 2.2, applied to the pair $(\mu, \nu)=(|\sigma|,|\sigma|)$, yields (8). In particular, $|\sigma|$ is bounded. Together with 5.3 applied once to $\psi(t)=\varphi_{0}\left(t^{2}\right)$ and once to $\psi(t)=(-)^{k} \varphi_{B}\left(t^{2}\right)$, it follows that $M^{\varphi}\left(\mathbb{R}^{d}\right)$ is the intersection of three vector subspaces of $M\left(\mathbb{R}^{d}\right)$ (two as in 5.3, the remaining one being the space of all bounded measures), and that

$$
I(\varphi ; \cdot, \cdot)=I\left(\varphi_{0} ; \cdot, \cdot\right)+I\left(\varphi_{A} ; \cdot, \cdot\right)+I\left(\varphi_{B} ; \cdot, \cdot\right)
$$

is the sum of three well-defined real-valued bilinear forms on $M^{\varphi}\left(\mathbb{R}^{d}\right)$.

The strict positive definiteness on the indicated subspace is a consequence of Corollary 2.4.

6.7. Proof of Corollary 2.6. We may assume $\varphi \in \mathcal{B}_{k} \backslash \mathcal{B}_{k-1}$. Call the set in question $M_{c}^{\varphi}$. By Corollary 2.5 and the linearity of $\sigma \mapsto \int x^{\alpha} d \sigma(x), M_{c}^{\varphi}$ is in fact convex. For $\sigma, \tau \in M_{c}^{\varphi}$ with $\sigma \neq \tau$ and for $\lambda \in(0,1)$ we have, using Corollary 2.5 again,

$$
\begin{aligned}
& \lambda \cdot I(\varphi ; \sigma, \sigma)+(1-\lambda) \cdot I(\varphi ; \sigma, \sigma)-I(\varphi ; \lambda \sigma+(1-\lambda) \tau, \lambda \sigma+(1-\lambda) \tau) \\
& =\lambda \cdot(1-\lambda) \cdot I(\varphi ; \sigma-\tau, \sigma-\tau) \\
& >0 . \quad \square
\end{aligned}
$$

\section{ACKNOWLEDGEMENT}

I thank Paul Ressel for drawing my attention to Guo, Hu \& Sun [11], and Karsten Müller for an idea leading to the present form of 5.1.

\section{REFERENCES}

[1] R. Alexander and K.B. Stolarsky, Extremal problems of distance geometry related to energy integrals. Trans. Amer. Math. Soc. 193 (1974), 1-31. MR 50:3121

[2] C. Berg, J. P. R. Christensen and P. Ressel, Harmonic Analysis on Semigroups. Theory of Positive Definite and Related Functions. Springer, New York, 1984. MR 86b:43001

[3] A. S. Besicovitch, A general form of the covering principle and relative differentiation of addititive functions. Proc. Cambridge Philos. Soc. 41 (1945), 103-110. MR 7:10e

[4] R. N. Bhattacharya and R. Ranga Rao, Normal Approximation and Asymptotic Expansions. Wiley, New York, 1986. MR 87k:60062; MR 55:9219

[5] G. Björk, Distributions of positive masses which maximize a certain generalized energy integral. Ark. Mat. 3 (1956), 255-269. MR 17:1198b 
[6] A. Buja, B. F. Logan, J. A. Reeds and L. A. Shepp, Inequalities and positive-definite functions arising from a problem in multidimensional scaling. Ann. Statist. 22 (1994), 406-438. MR 95a:60021

[7] J. L. Doob, Classical Potential Theory and Its Probabilistic Counterpart. Springer, New York, 1984. MR 85k:31001

[8] C. G. Esseen, Bounds for the absolute third moment. Scand. J. Statist. 2 (1975), 149-152. MR 52:4376

[9] W. Feller, An Introduction to Probability Theory and Its Applications. Vol. 2, Second Edition. Wiley, New York, 1971. MR 42:5292

[10] O. Frostmann, Potentiel d'équilibre et capacité des ensemble avec quelques applications a la théorie des fonctions. Thèse, Lund. Meddelanden Lunds Univ. Mat. Sem. 31935.

[11] K. Guo, S. Hu and X. Sun, Conditionally positive definite functions and Laplace-Stieltjes integrals. J. Approx. Theory 74 (1993), 249-265. MR 94k:26015

[12] E. Hille, Analytic Function Theory. Vol. 2. Blaisdell Publishing Company, Waltham, Massachusetts, 1962. MR 34:1490

[13] —, Methods in Classical and Functional Analysis. Addison-Wesley, Reading, Massachusetts, 1972. MR 57:3802

[14] W. C. M. Kallenberg, Problem 319. Statistica Neerlandica 49 (1995), 132.

[15] N. S. Landkof, Foundations of Modern Potential Theory. Springer, Berlin, 1972. MR 50:2520

[16] L. Mattner, Behandlung einiger Extremalprobleme für Wahrscheinlichkeitsverteilungen. Dissertation, Universität Hannover, 1990.

[17] Completeness of location families, translated moments, and uniqueness of charges. Probab. Theory Related Fields 92 (1992), 137-149. MR 93f:60024

[18] _ Extremal problems for probability distributions: a general method and some examples. In: Stochastic Inequalities, Moshe Shaked and Y. L. Tong, eds., IMS Lecture NotesMonograph Series Vol. 22, Inst. Math. Statist, Hayward, CA, 1992, pp. 224-283. MR 94c:60028

[19] _ Bernstein's Theorem, inversion formula of Post and Widder, and the uniqueness theorem for Laplace transforms. Expo. Math. 11 (1993), 137-140. MR 94b:44001

[20] C. A. Micchelli, Interpolation of scattered data: distance matrices and conditionally positive definite functions. Constr. Approx. 2 (1986), 11-22. MR 88d:65016

[21] G. Polya and G. Szegö, Über den transfiniten Durchmesser (Kapazitätskonstante) von ebenen und räumlichen Punktmengen. J. Reine Angew. Math. 165 (1931), 4-49. Reprinted and commented on in: G. Polya (1974), Collected Papers, Vol. 1, Singularities of Analytic Functions, M.I.T. Press, Cambridge, Mass., and in: Gabor Szegö (1982), Collected Papers, Vol. 2., Birkhäuser, Boston. MR 58:21341; MR 84d:01082b

[22] M. Riesz, Intégrales de Riemann-Liouville et potentiels. Acta Szeged Sect. Math. 9 (1937), 1-42. Reprinted in: Marcel Riesz (1988), Collected Papers, Springer, Berlin, pp. 482-523. MR 90a:01109

[23] W. Rudin, Representation of functions by convolutions. J. Math. and Mech. 7 (1959), 103116. MR 21:3728

[24] _ Real and Complex Analysis, Second Edition. McGraw-Hill, New York (1974). MR 49:8783

[25] Z. Sasvári, Positive Definite and Definitizable Functions. Akademie Verlag, Berlin, 1994. MR 95c: 43005

[26] I. J. Schoenberg, Metric spaces and completely monotone functions. Ann. Math. 39 (1938), 811-841. Reprinted in: I. J. Schoenberg (1988), Selected Papers. Vol. 1. Birkhäuser, Boston, pp. 115-145. MR 91c:01051a

Universität Hamburg, Institut für Mathematische Stochastik, Bundesstr. 55, D20146 Hamburg, Germany

E-mail address: mattner@math.uni--hamburg.de 